\title{
Assessing the outcomes of everolimus on renal angiomyolipoma associated with tuberous sclerosis complex in China: a two years trial
}

Yi Cai ${ }^{1 \dagger}$, Hao Guo ${ }^{1 \dagger}$, Wenda Wang ${ }^{1 \dagger}$, Hanzhong $\mathrm{Li}^{1}$, Hao Sun ${ }^{2}$, Bing Shi ${ }^{2}$ and Yushi Zhang ${ }^{1 *}$

\begin{abstract}
Background: Tuberous sclerosis complex (TSC) is a rare autosomal dominant genetic disorder characterized by the development of numerous benign tumors. Renal angiomyolipoma (RAML) occur in up to $80 \%$ of TSC patients, which is a leading cause of TSC-related death in adult patients. The aim of the study was to evaluate the efficacy and safety profiles of everolimus in Chinese patients of TSC associated with RAML(TSC-RAML).
\end{abstract}

Methods: In this 2-years, nonrandomized, open-label trial, 18 patients of TSC-RAML, with at least one RAML $3 \mathrm{~cm}$ or larger in its longest diameter, were enrolled to assess the efficacy and safety of everolimus therapy in Chinese patients. Everolimus was administered for the first 12 months only. The primary endpoint was a reduction of 50\% or more relative in RAML volume to the baseline in the absence of new RAML $\geq 1 \mathrm{~cm}$ and no RAML-related bleeding of grade $\geq 2$. The secondary endpoints included: safety, lung function and skin lesions response rate. Serial computed tomography of RAML, magnetic resonance imaging of brain lesions and pulmonary-function tests were performed. Adverse events were investigated using CTCAE v4.0. All analyses used a significance level of 0.05 and were generated in SPSS19.0 software.

Results: The proportion of patients who achieved $\geq 50 \%$ reduction from baseline in the sum of volumes of target lesions increased from $52.94 \%$ at 3 months, to $58.82 \%$ and $66.67 \%$ at months 6 and 12, respectively. During the period of everolimus therapy, among patients with lymphangioleiomyomatosis, the mean forced expiratory volume in $1 \mathrm{~s}$ (FEV1) increased by $276 \pm 78 \mathrm{ml}(P<0.001)$, the forced vital capacity $(F V C)$ increased by $433 \pm 170 \mathrm{ml}$ $(P<0.001)$, and the residual volume decreased by $408 \pm 243 \mathrm{ml}(P=0.009)$, as compared with baseline values. The angiomyolipoma volume and the lung function approached, but did not completely return to, the baseline values. The skin lesions response rate was $37.5 \%$ after 12 months of therapy falling to $21.4 \%$ at 12 months after stopping everolimus. The most common adverse events were mucositis oral, irregular menstruation, abdominal pain, hypertriglyceridemia and headache. The most common grade 3 adverse events were irregular menstruation and mucositis oral. In addition, one patient died from RAML spontaneous haemorrhage during treatment with everolimus, even with reduction in RAML volume of $60.68 \%$ at 3 months. A second death was due to epithelioid RAML progression, with metastasis to multiple retroperitoneal lymph node, who died from severe infection one month after surgery.

\footnotetext{
* Correspondence: zhangyushi2014@126.com

${ }^{\dagger}$ Equal contributors

${ }^{1}$ Department of Urology, Peking Union Medical College Hospital, Chinese

Academy of Medical Sciences and Peking Union Medical College, 1

Shuaifuyuan Road, Beijing 100730, China

Full list of author information is available at the end of the article
} 
(Continued from previous page)

Conclusions: Angiomyolipomas regressed somewhat during everolimus therapy but tended to increase in volume after the therapy was stopped. Everolimus was well tolerated and showed promising activity in Chinese patients with TSC-RAML, however, we should alert the life-threatening hemorrhage of large RAML in the early period and the lymph node metastasis of epithelioid RAML.

Trial registration: ChiCTR-OPC-14005488. Registered November 17, 2014.

Keywords: Angiomyolipoma, Tuberous sclerosis complex, Everolimus, Treatment outcome, Safety

\section{Background}

Tuberous sclerosis complex (TSC) is an autosomal dominant syndrome affecting 1 2 million people worldwide [1]. It is characterized by prominent neurodevelopmental features and by tumors that develop in the brain, skin, heart, kidneys and lungs [1]. Renal angiomyolipoma (RAML) develop in approximately $80 \%$ of adults and adolescents with TSC $[1,2]$. RAML associated with tuberous sclerosis complex (TSC-RAML) are characterized as multiple and commonly bilateral lesions that consist of blood vessels, smooth muscles, and adipose tissues [2]. TSC-RAML typically grow over time, presenting of arterial hypertension and risk of potentially life-threatening hemorrhage, which is the leading cause of TSC-associated death in adult patients [3]. To date, the main therapeutic options are embolisation, elective surgery, and emergency nephrectomy in cases of uncontrollable haemorrhage in China [4].

The majority of individuals with TSC have mutations in either the TSC1 or TSC2 genes, and subsequent somatic mutation results in constitutive activation of mammalian target of rapamycin (mTOR), a critical regulator of cell growth, proliferation, and angiogenesis [5-7]. Bissler JJ and colleagues demonstrated everolimus, a mammalian target of rapamycin (mTOR) inhibitor, could significant reduction in renal angiomyolipoma volume compared with placebo in Western populations [8]. The International tuberous sclerosis complex consensus conference held in 2012 recommended mTOR inhibitors as the first-line treatment for RAML when enlarged to $3 \mathrm{~cm}$ or more, even when asymptomatic [9]. Transcatheter arterial embolization and partial nephrectomy are recommended as second-line treatments [9]. However, we lack data of the efficacy and safety of everolimus in treatment of TSC-RAML in Chinese patients.

The objective of the present study was to evaluate the efficacy and safety of everolimus for TSC-RAML in Chinese patients.

\section{Methods}

\section{Patients}

This trial was a 2-years, nonrandomized, open-label trial, phase 2 study (ChiCTR-OPC-14005488, https://www. chictr.org.) conducted at Peking Union Medical College Hospital start from December 2014. From December
2014 to November 2015, patients were included if they met the following inclusion criteria: (1). Men or women (not pregnant) $\geq 18$ years; (2). Clinical and/or genetic diagnosis of TSC; (3). CT or MRI shows one or more TSCRAMLs with the longest diameter $\geq 3 \mathrm{~cm}$; (4). Without RAML bleeding or embolism in the past 6 months. Patients were excluded from the study if they met the following exclusion criteria: (1). Age $<18$ years; (2). Women who plan to be pregnant or have been pregnant or lactating; (3). CT or MRI shows the longest diameter of RAML $<3 \mathrm{~cm}$; (4). Patients expected to undergo surgery or embolization therapy during the trial; (5). History of coronary heart disease, myocardial infarction or cerebral infarction related to atherosclerosis; (6). History of RAML bleeding or embolism in the past 6 months; (7). Impaired lung function defined as following: For patients without lymphangioleiomyomatosis (LAM): Known impaired lung function (e.g. FEV1 or DLco $\leq 70 \%$ of predicted); For patients with LAM: DLco $\leq 35 \%$, or $\mathrm{O}_{2}$ saturations below normal at rest, or $\mathrm{O}_{2}$ saturation $\leq 88 \%$ on 6 min walking test with up to $6 \mathrm{l} \mathrm{O}_{2} / \mathrm{min}$ nasal oxygen; (8). Severe hematological disease or liver function abnormality (such as aminotransferase $>2.5$ times of normal upper limit, serum bilirubin $>1.5$ times of normal upper limit, hemoglobin $<9 \mathrm{~g} / \mathrm{dL}$, platelet $<80,000 / \mathrm{mm}^{3}$, or absolute neutrophil count $<1000 / \mathrm{mm}^{3}$ ); (9). Concomitant severe infection before or during the trial; (10). Previous organ transplantation; (11). History of other surgeries (involving entry into body cavity or suture) in the past 2 months; (12). Previous mTOR inhibitor treatment (such as sirolimus and everolimus); (13). Use of investigational drug within 30 days; (14). Poor control of hyperlipidemia: fasting serum cholesterol $>300 \mathrm{mg} / \mathrm{dL}$ (or $>7.75 \mathrm{mmol} / \mathrm{L}$ ), fasting triglyceride $>2.5$ times of normal upper limit; (15). Poor control of diabetes: fasting blood glucose $>1.5$ times of normal upper limit; (16). Patients with bleeding tendency or using oral anti-vitamin $\mathrm{K}$ drugs (except low-dose warfarin); (17). History of HIV seropositivity; (18). Active hepatitis; (19). Patients who cannot participate in regular visits and follow-up; (20). Patients who are not suitable for MRI examination (such as excessive obesity, mental disorders, bullet fragment in body, stent and pacemaker); (21). Serum creatinine $>1.5$ times of normal upper limit. This study protocol was approved by the Human Ethics Committee of 
Peking Union Medical College Hospital, before the first patient was enrolled.

\section{Study design}

This study includes two periods: the core period and the extended period. Everolimus was administered for the first 12 months only. The core period of the study lasts for 1 year, and then all possible patients will continue the next 1 -year extended observation. The dose is initiated with $10 \mathrm{mg}$ daily by oral, and then titrated to the target tough blood concentration of $5-15 \mathrm{ng} / \mathrm{ml}$ during the first 3 months and maintained throughout the observation, with dose modifications allowed on the basis of safety findings. Concomitant use of strong inhibitors or inducers of cytochrome P450 3A4 or p-glycoprotein (PgP) was to be avoided during the study; use of antiproliferative agents other than study drug was prohibited. All planed visits and assessments are listed in Additional file 1: Table S1. The primary efficacy endpoint was the proportion of patients with a confirmed angiomyolipoma response, defined as a reduction in angiomyolipoma volume (sum of volumes of all target angiomyolipomas $>1 \mathrm{~cm}$ identified at baseline) of $50 \%$ or more relative to baseline and absence of angiomyolipoma progression. In addition, RAML response requires satisfying the following criteria: (1) No new RAML $\geq 1.0 \mathrm{~cm}$ in longest diameter are identified; (2) The patient does not have any RAML-related bleeding of grade $\geq 2$ as defined by the National Cancer Institute Common Terminology Criteria for Adverse Events, version 4.0 (NCI-CTCAE v.4). Key secondary endpoints were time to angiomyolipoma progression and skin lesions response rate. Computed Tomography (CT) or magnetic resonance imaging(MRI), same modality used throughout the study for each patient, used to calculate RAMLs volume at baseline and repeated at 3, 6, 12, 18 and 24 months after the start of treatment. Skin lesions resulting from tuberous sclerosis complex include hypomelanotic macules, the shagreen patch, periungual or subungual fibromas, and facial angiofibromas, forehead plaques, or both, and were assessed at baseline and repeated at 3, 6, 12, 18 and 24 months after the start of treatment using the sevenpoint grading scale Physician's Global Assessment of Clinical Condition [10,11] (Additional file 2: Table S2). Patients with lymphangioleiomyomatosis underwent pulmonaryfunction testing at baseline, 12 months, and 24 months. Adverse events were monitored throughout the study and graded according to the Common Terminology Criteria for Adverse Events v4.0 via patient-reported or caregiverreported responses as well as investigator assessment.

\section{Statistical analysis}

Data are expressed as the mean \pm standard deviation $(\mathrm{M} \pm$ $\mathrm{SD})$ or $\mathrm{n}(\%)$. Statistical significance was determined by paired or unpaired Student's t-test in cases of standardized expression data. The SPSS software package (version 17.0) was used for all statistical analysis. $P$ value $<0.05$ was defined as statistically difference.

\section{Results}

\section{Characteristics of the patients}

Eighteen patients were enrolled in this trial. Their demographic details and disease characteristics are summarized in Table 1. The median age was 29 years, 9 patients were under 30 years of age. All 18 patients underwent genotyping by next-generation sequencing and confirmed all for TSC2 mutation (Additional file 3: Table S3). Six female patients were diagnosed for lymphangioleiomyomatosis (LAM) while one patient presence of subependymal giant cell astrocytoma. Two patients had undergone embolisation, and two patients had previously undergone partial

Table 1 Baseline patient demographic and disease characteristics

\begin{tabular}{|c|c|}
\hline & Everlimus $(N=18)$ \\
\hline Age in years, median (range) & $29(20 \sim 46)$ \\
\hline$<30$ year & $9(50 \%)$ \\
\hline$\geq 30$ year & $9(50 \%)$ \\
\hline \multicolumn{2}{|l|}{ Sex } \\
\hline Women & $12(66.7 \%)$ \\
\hline Men & $6(33.3 \%)$ \\
\hline \multicolumn{2}{|l|}{ Gene mutation } \\
\hline TSC1 & $0(0 \%)$ \\
\hline TSC2 & $18(100 \%)$ \\
\hline Diagnosis of lymphangioleiomyomatosis & $6(33.3 \%)$ \\
\hline Skin lesion $(\geq 1)$ & 18(100\%) \\
\hline Presence of subepedymal giant cell astrocytoma & $1(5.6 \%)$ \\
\hline \multicolumn{2}{|c|}{ Longest diameter of the largest renal angiomyolipoma lesion } \\
\hline$\geq 8 \mathrm{~cm}$ & $14(77.8 \%)$ \\
\hline$\geq 4 \mathrm{~cm}$ and $<8 \mathrm{~cm}$ & $4(22.2 \%)$ \\
\hline$<4 \mathrm{~cm}$ & $0(0 \%)$ \\
\hline \multicolumn{2}{|c|}{ Sum of volumes of target renal angiomyolipoma lesions, $\mathrm{cm}^{3}$} \\
\hline Mean (SD) & $1974(2406)$ \\
\hline Median (range) & $1247(60.77 \sim 9079)$ \\
\hline Bilateral angiomyolipoma & 16(88.9\%) \\
\hline \multicolumn{2}{|l|}{ Number of target angiomyolipoma lesions } \\
\hline $1 \sim 2$ & $11(61.1 \%)$ \\
\hline $3 \sim 4$ & $7(38.9 \%)$ \\
\hline \multicolumn{2}{|l|}{ Previous angiomyolipoma therapy } \\
\hline Surgery/invasive procedure & $6(33.3 \%)$ \\
\hline Renal embolisation & $2(11.1 \%)$ \\
\hline Partial nephrectomy & $2(11.1 \%)$ \\
\hline Nephrectomy & $2(11.1 \%)$ \\
\hline Medication & $0(0 \%)$ \\
\hline
\end{tabular}


nephrectomy while another two undergone unilateral nephrectomy. In addition, there were no large, at least $5 \mathrm{~mm}$ or larger in size, intra-renal aneurysm in all 18 patients. Three patients left the study during the first year: one had a unilateral renal hemorrhage and died at fourth fellow-up month, one died due to epithelioid RAML progression at eleven fellow-up month, and one did not comply with the protocol. Fifteen patients underwent the 12 -month evaluation. One withdrew from the study after the 18-month visit to continue everolimus therapy off-label because of worry about tumor progress fast, leaving 14 patients at the 24-month assessment of angiomyolipoma.

\section{Treatment efficacy}

The proportion of patients who achieved $\geq 50 \%$ reduction from baseline in the sum of volumes of target lesions increased from $52.94 \%(9 / 17)$ at 3 months, to 58 . $82 \%(10 / 17)$ and $66.67 \%(10 / 15)$ at months 6 and 12, respectively. Primary-tumor shrinkage was most rapid during the initial 3 months of treatment, with evidence of a sustained response at subsequent time points during the core treatment phase. Median time to angiomyolipoma response for everolimus was 3.0 months. The mean renal angiomyolipoma volume at baseline was $1974 \pm$ $2406 \mathrm{ml}$ (Table 1). After 12 months of therapy, the mean volume decreased to $41.14 \pm 26.54 \%$ of the baseline volume $(P<0.002)$ (Table 2 and Fig. 1$)$. At 6 and 12 months after stopping everolimus, the mean angiomyolipoma volume had increased to $60.67 \pm 23.28 \%$ of the baseline volume $(P=0.006)$ and $77.62 \pm 16.66 \%$ of the baseline volume $(P=0.014)$, respectively (Table 2 and Fig. 1$)$. The response of a renal angiomyolipoma along with time of everolimus therapy, visualized on CT, is shown in Fig. 2. The current trial shows that everolimus are effective in reducing angiomyolipoma size in tuberous sclerosis in Chinese patients.

Pulmonary functional data for 6 female patients with lymphangioleiomyomatosis are listed in Table 3. All six patients had never been smokers. At enrollment, spirometric measurements were normal in one patients, revealed moderate airflow obstruction (forced expiratory volume in $1 \mathrm{~s}$ [FEV1], 50 to $70 \%$ of the predicted value) in three patients, and indicated severe airflow obstruction (FEV $1<50 \%$ of the predicted value) in two patients. During everolimus therapy, the mean FEV1 increased

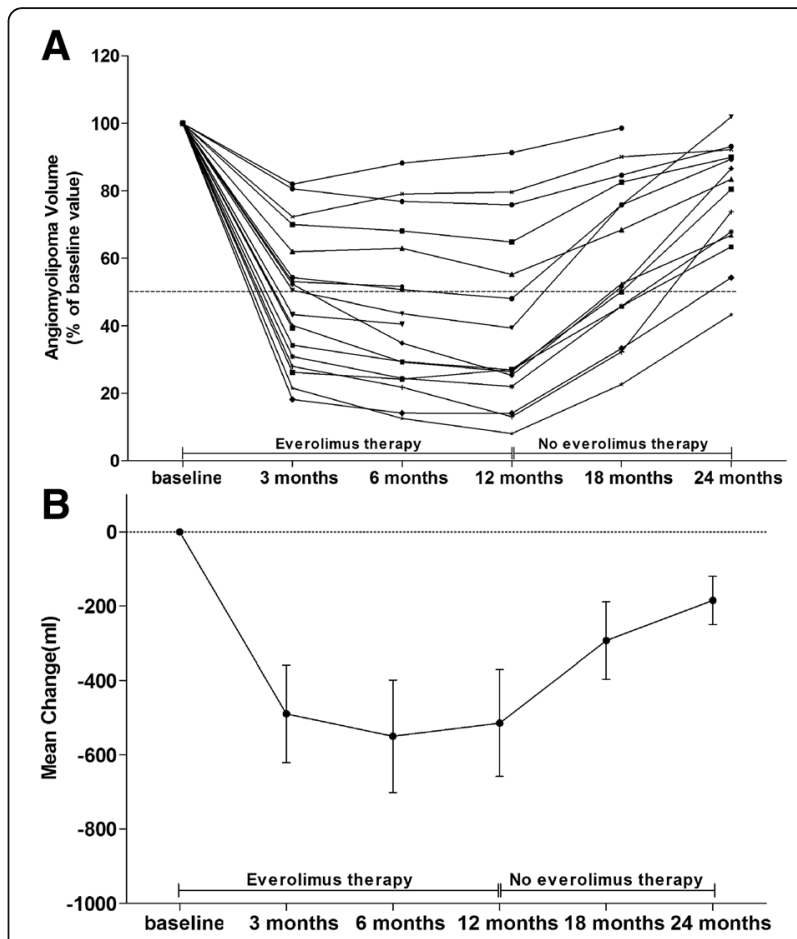

Fig. 1 Renal Angiomyolipoma Volume in the Patients with the Tuberous Sclerosis Complex during the Study. Panel a shows the renal angiomyolipoma volume at each visit is expressed as a percentage of the baseline size. The dashed line represents $50 \%$ of the baseline value; data below the line indicate that the mean angiomyolipoma volume was reduced by $50 \%$ or more. Panel $\mathbf{b}$ shows the mean change (in milliliters) from the baseline values for renal angiomyolipoma volume. I bars indicate the standard errors

from the baseline mean by $276 \pm 78 \mathrm{ml}$ at 12 months $(P<0.001)$, while the mean FVC increased from the baseline mean by $433 \pm 170 \mathrm{ml}$ at 12 months $(P<0.001)$ (Fig. 3a and c). After 1 year of everolimus therapy, the FEV1 and FVC in these patients were significantly improved (Table 3). Twelve months after stopping everolimus, the mean FEV1 was $126 \pm 48 \mathrm{ml}$ greater than the mean baseline value $(P=0.004)$, while the mean FVC was $274 \pm 142 \mathrm{ml}$ greater than the mean baseline value $(P=0.008)$ (Fig. $3 \mathrm{~b}$ and $\mathrm{c})$. The mean percent of the predicted FEV1 value were significantly improved at 12 months $(P<0.001)$ and at 24 months $(P=0.008)$ (Table 3). The mean percent of the predicted FVC value were significantly improved at 12 months $(P<0.001)$ and

Table 2 Response of angiomyolipoma volume to everolimus therapy

\begin{tabular}{|c|c|c|c|c|c|}
\hline & 3 months & 6 months & 12 months & 18 months & 24 months \\
\hline Patients (n) & 18 & 17 & 15 & 15 & 14 \\
\hline No. of response $(n, \%)$ & $9(52.94 \%)$ & $10(58.82 \%)$ & $10(66.67 \%)$ & $5(33.33 \%)$ & $1(7.14 \%)$ \\
\hline${ }^{a_{0}} \%$ of baseline value (Mean $\pm S D, \%$ ) & $47.73 \pm 19.81$ & $44.27 \pm 23.69$ & $41.14 \pm 26.54$ & $60.67 \pm 23.28$ & $77.62 \pm 16.66$ \\
\hline$P$ value for change from baseline value & 0.002 & 0.002 & $<0.001$ & 0.006 & 0.014 \\
\hline
\end{tabular}

athe average percentage change of baseline in the total volume of all target AML lesions 

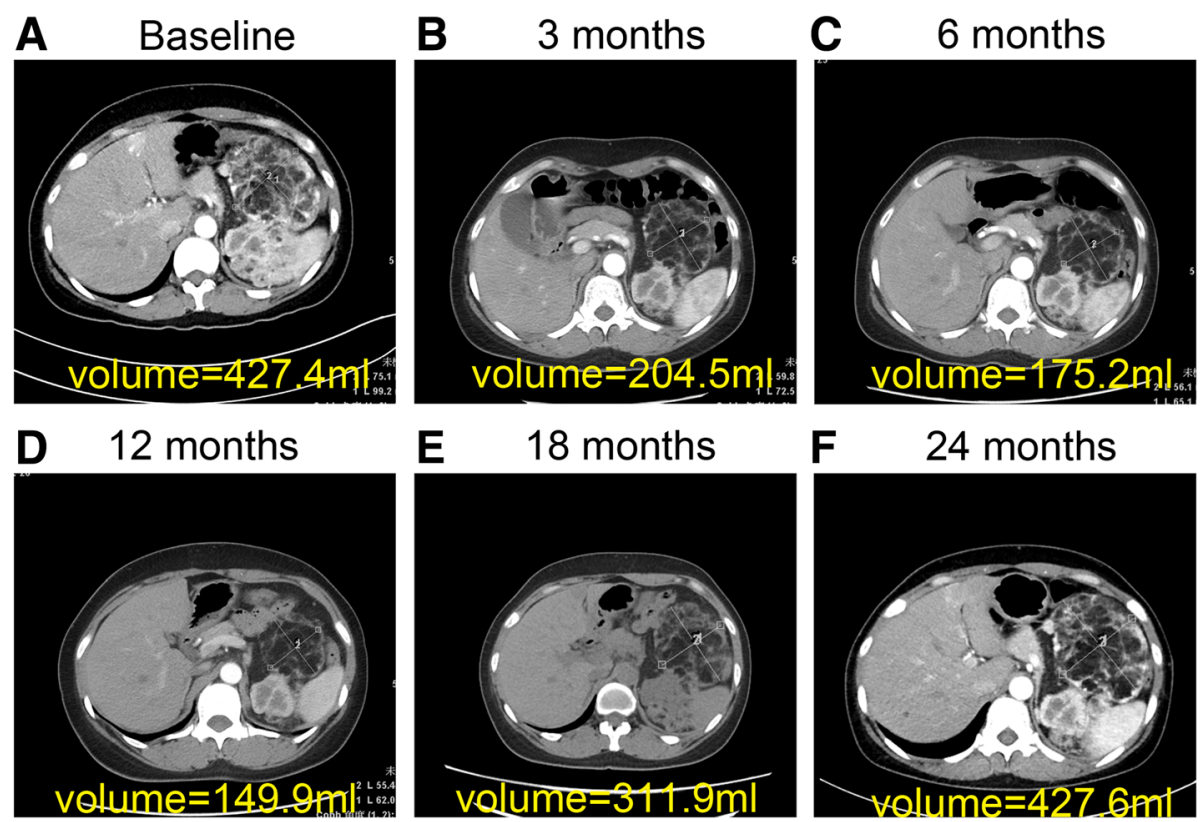

Fig. 2 Target Renal Angiomyolipomas of a Patient with the Tuberous Sclerosis Complex. Panel a shows the target renal angiomyolipoma volume (in milliliters) at baseline. Panel $\mathbf{b}, \mathbf{c}$ and $\mathbf{d}$ shows the target renal angiomyolipoma volume (in milliliters) after 3, 6 and 12 months of everolimus therapy. Panel $\mathbf{e}$ and $\mathbf{f}$ shows the target renal angiomyolipoma volume (in milliliters) at 6 and 12 months after stopping everolimus

at 24 months $(P<0.001)$ (Table 3$)$. The mean residual volume fell by $408 \pm 243 \mathrm{ml}$ after 1 year of everolimus therapy, as compared with the baseline value $(P=0.009)$ (Table 3$)$. The mean percent of the predicted residual volume were significantly improved at 12 months $(P<0.001)$ and at 24 months $(P<0.001)$ (Table 3$)$. Neither the DLco nor total lung capacity changed significantly during the study (Table 3).

All patients had cortical tubers; 15 patients had subependymal nodules and one had subependymal giant-cell

Table 3 Pulmonary Structural and Functional Characteristics of Patients with Lymphangioleiomyomatosis

\begin{tabular}{|c|c|c|c|c|c|}
\hline \multirow[t]{2}{*}{ Value } & \multirow{2}{*}{$\begin{array}{l}\text { Baseline } \\
(n=6)\end{array}$} & \multirow{2}{*}{$\begin{array}{l}12 \text { months } \\
(n=6)\end{array}$} & \multirow{2}{*}{$\begin{array}{l}24 \text { months } \\
(n=5)\end{array}$} & \multicolumn{2}{|l|}{ Change from Baseline } \\
\hline & & & & 12 months $(n=6)$ & 24 months $(n=5)$ \\
\hline \multicolumn{6}{|l|}{ FEV1 } \\
\hline Least-square mean — liters & $1.59 \pm 0.41$ & $1.87 \pm 0.47$ & $1.65 \pm 0.45$ & $0.28 \pm 0.08(P<0.001)$ & $0.13 \pm 0.05(P=0.004)$ \\
\hline Percent of predicted value & $58.51 \pm 11.09$ & $65.32 \pm 12.32$ & $59.89 \pm 11.58$ & $6.81 \pm 2.28(P<0.001)$ & $3.46 \pm 1.57(P=0.008)$ \\
\hline \multicolumn{6}{|l|}{ FVC } \\
\hline Least-square mean — liters & $2.77 \pm 0.56$ & $3.20 \pm 0.68$ & $2.95 \pm 0.66$ & $0.43 \pm 0.17(P=0.002)$ & $0.27 \pm 0.14(P=0.013)$ \\
\hline Percent of predicted value & $79.90 \pm 12.68$ & $87.16 \pm 12.63$ & $84.36 \pm 13.71$ & $7.26 \pm 2.16(P<0.001)$ & $6.39 \pm 1.36(P<0.001)$ \\
\hline \multicolumn{6}{|l|}{ Total lung capacity } \\
\hline Least-square mean — liters & $5.16 \pm 0.51$ & $5.18 \pm 0.44$ & $5.14 \pm 0.49$ & $0.03 \pm 0.12(P=0.62)$ & $0.04 \pm 0.10(P=0.43)$ \\
\hline Percent of predicted value & $99.21 \pm 7.01$ & $99.70 \pm 7.28$ & $97.75 \pm 7.24$ & $0.49 \pm 1.47(P=0.45)$ & $-0.22 \pm 0.78(P=0.57)$ \\
\hline \multicolumn{6}{|l|}{ Residual volume } \\
\hline Least-square mean — liters & $2.39 \pm 0.10$ & $1.98 \pm 0.31$ & $2.11 \pm 0.29$ & $-0.41 \pm 0.24(P=0.009)$ & $-0.31 \pm 0.23(P=0.036)$ \\
\hline Percent of predicted value & $124.56 \pm 10.04$ & $104.23 \pm 8.85$ & $112.30 \pm 5.41$ & $-20.32 \pm 1.62(P<0.001)$ & $-15.79 \pm 2.38(P<0.001)$ \\
\hline \multicolumn{6}{|l|}{ DLCO } \\
\hline Least-square mean $-\mathrm{ml} / \mathrm{mmHg} / \mathrm{min}$ & $12.93 \pm 2.42$ & $13.00 \pm 2.41$ & $12.59 \pm 2.39$ & $0.07 \pm 0.46(P=0.71)$ & $-0.21 \pm 0.55(P=0.43)$ \\
\hline Percent of predicted value & $53.40 \pm 9.29$ & $53.60 \pm 8.70$ & $54.27 \pm 9.56$ & $0.19 \pm 1.39(P=0.74)$ & $1.68 \pm 2.11(P=0.15)$ \\
\hline
\end{tabular}

Data are listed for 6 patients with lymphangioleiomyomatosis who completed the 12-month study visit. Data for the 24-month study visit were available for 5 of these 6 patients; 1 patient had withdrawn during the second year. Changes from baseline were not significant unless otherwise indicated. DLco denotes diffusing capacity of the lung for carbon monoxide, FEV1 forced expiratory volume in $1 \mathrm{~s}$, FVC forced vital capacity 

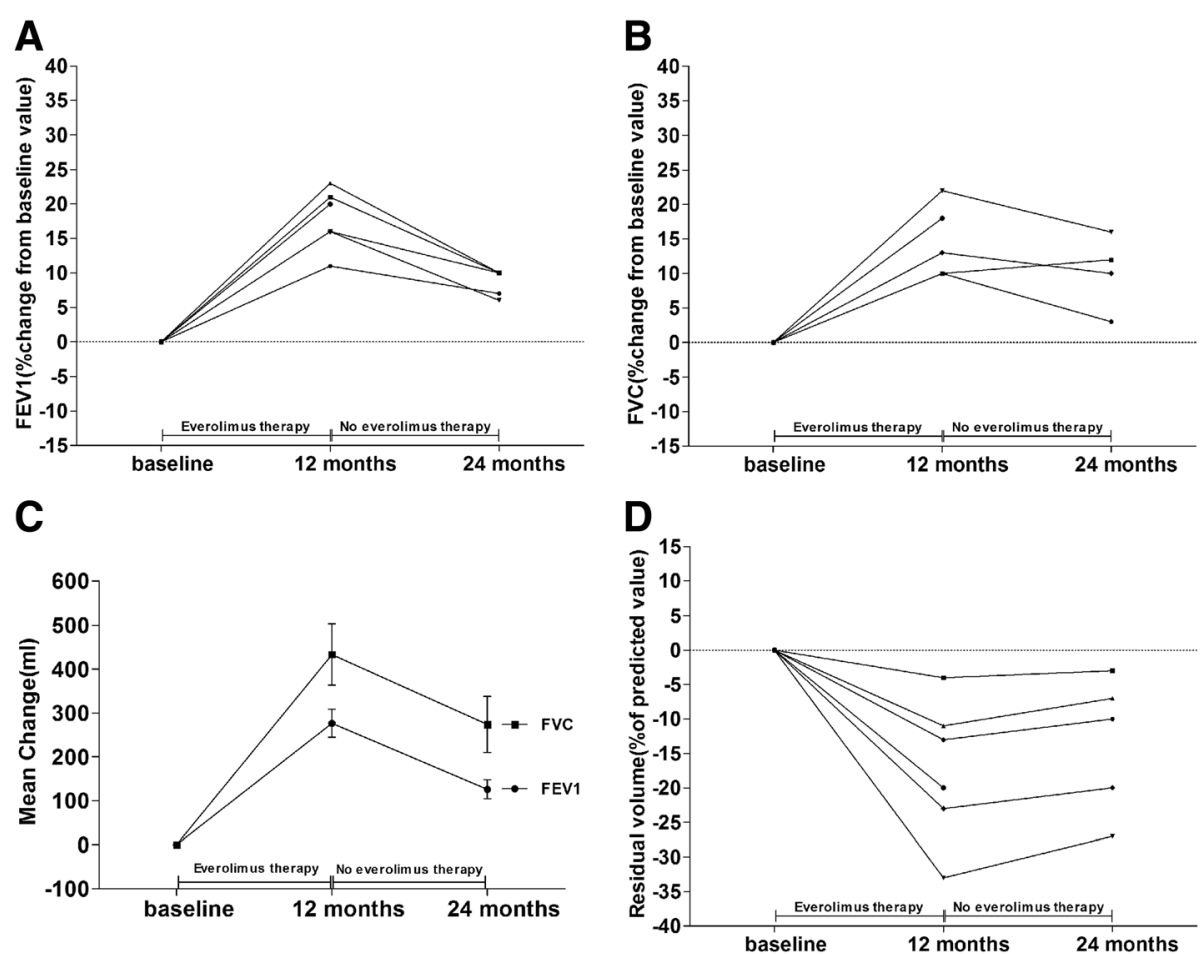

Fig. 3 Pulmonary Function in Patients with Lymphangioleiomyomatosis. Panel a shows the forced expiratory volume in $1 \mathrm{~s}$ (FEV1) for each patient. Panel $\mathbf{b}$ shows the forced vital capacity (FVC) for each patient. Panel $\mathbf{c}$ shows the mean change (in milliliters) from the baseline values for FEV1 and for FVC. I bars indicate the standard errors. Panel $\mathbf{d}$ shows the residual volume for each patient

astrocytomas. There were no changes in the size of the subependymal nodules. We did not get the evaluable data of the patient with subependymal giant-cell astrocytomas as she died for RAML spontaneous haemorrhage in four months. Although this was not a study endpoint, a slight improvement in the frequency of seizures was reported by some parents.
Skin lesions associated with tuberous sclerosis were present at baseline in all 18 patients. Facial angiofibromas decreased in size and became paler and less rough after 12 months of therapy (Fig. 4). The skin lesions response rate was $37.5 \%$ (6 of 16) after 12 months of therapy falling to $21.4 \%$ ( 3 of 14 ) at 12 months after stopping everolimus.
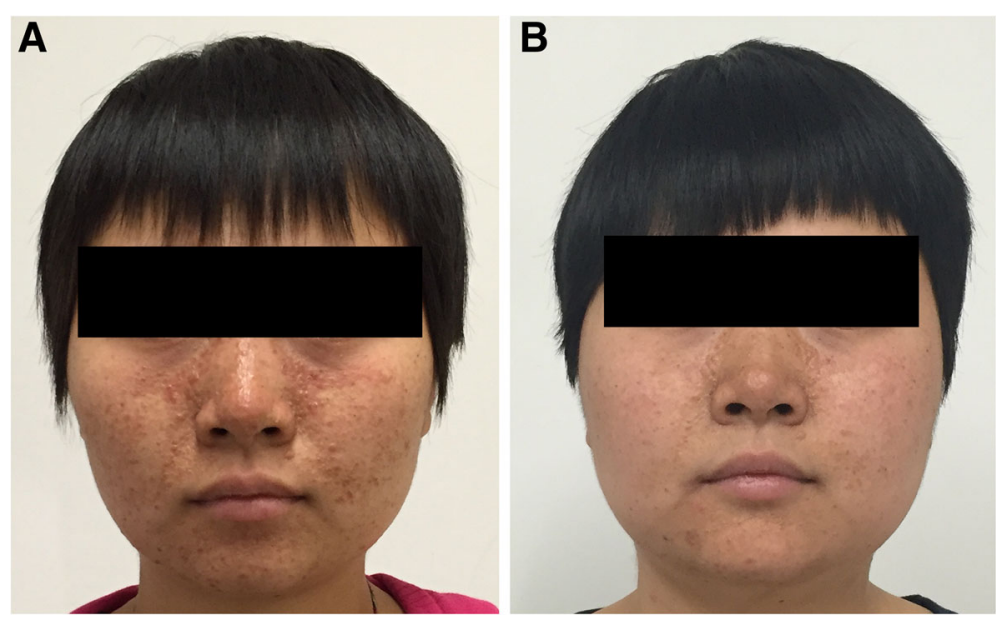

Fig. 4 Facial angiofibromas of a Patient with the Tuberous Sclerosis Complex. Panel a shows the facial angiofibromas at baseline. Panel $\mathbf{b}$ shows the facial angiofibromas improvement after 12 months of everolimus therapy 


\section{Adverse events}

Adverse events were consistent with the known everolimus safety profile. The most common adverse events were mucositis oral (100\%), irregular menstruation (91. $7 \%)$, abdominal pain (77.8\%), hypertriglyceridemia (72. $2 \%)$ and headache $(66.7 \%)$ (Table 4$)$. The most common grade 3 adverse events were irregular menstruation (25\%) and mucositis oral (11.1\%) (Table 4). Three patients with irregular menstruation was classified as grade 3 adverse events due to persistent amenorrhea for more than 6 months. In the trial, one patient died from RAML spontaneous haemorrhage during treatment with everolimus, even with reduction in RAML volume of $60.68 \%$ at 3 months. A second death was due to epithelioid RAML progression, who underwent left radical nephrectomy and immunohistochemical examination revealed features of epithelioid AML accompany with retroperitoneal multiple

Table 4 Adverse events by preferred term, regardless of relationship to everolimus

\begin{tabular}{|c|c|c|c|c|}
\hline \multirow[t]{2}{*}{$\overline{n(\%)}$} & \multirow[b]{2}{*}{ All grades } & \multicolumn{3}{|c|}{ Everolimus $(N=18)$} \\
\hline & & Grade 3 & Grade 4 & Grade 5 \\
\hline Mucositis oral & $18(100 \%)$ & $2(11.1 \%)$ & - & - \\
\hline Irregular menstruation & $11(91.7 \%)$ & $3(25.0 \%)$ & - & - \\
\hline Abdominal pain & $14(77.8 \%)$ & $1(5.6 \%)$ & - & - \\
\hline Hypertriglyceridemia & $13(72.2 \%)$ & - & - & - \\
\hline Headache & $12(66.7 \%)$ & - & - & - \\
\hline Diarrhea & $11(61.1 \%)$ & - & - & - \\
\hline Upper respiratory infection & $10(55.6 \%)$ & - & - & - \\
\hline Proteinuria & $9(50 \%)$ & $1(5.6 \%)$ & - & - \\
\hline Malaise & $9(50 \%)$ & - & - & - \\
\hline Rash acneiform & $8(44.4 \%)$ & - & - & - \\
\hline Cholesterol high & $8(44.4 \%)$ & - & - & - \\
\hline Fever & $6(33.3 \%)$ & - & - & - \\
\hline Urinary tract infection & $5(27.8 \%)$ & - & - & - \\
\hline Hematuria & $5(27.8 \%)$ & - & - & - \\
\hline Alkaline phosphatase increased & $5(27.8 \%)$ & - & - & - \\
\hline Constipation & $4(22.2 \%)$ & - & - & - \\
\hline GGT increased & $4(22.2 \%)$ & - & - & - \\
\hline Hypophosphatemia & $4(22.2 \%)$ & - & - & - \\
\hline Seizures & $3(16.7 \%)$ & - & - & - \\
\hline Pneumonitis & $2(11.1 \%)$ & $1(5.6 \%)$ & - & $1(5.6 \%)$ \\
\hline Vomiting & $2(11.1 \%)$ & $1(5.6 \%)$ & - & - \\
\hline Lymphocyte count decreased & $2(11.1 \%)$ & - & - & - \\
\hline Anemia & $2(11.1 \%)$ & - & - & - \\
\hline Renal hemorrhage & $1(5.6 \%)$ & - & - & $1(5.6 \%)$ \\
\hline Neutrophil count decreased & $1(5.6 \%)$ & - & - & - \\
\hline Hyperuricemia & $1(5.6 \%)$ & - & - & - \\
\hline Creatinine increased & $1(5.6 \%)$ & - & - & - \\
\hline
\end{tabular}

lymph node metastasis. The patient died from severe infection one month after surgery.

\section{Discussion}

In the current trial, we reported the efficacy and safety of everolimus in treatment of TSC-RAML in Chinese patients for the first time. Everolimus therapy in patients with the tuberous sclerosis complex was associated with a reduction in angiomyolipoma volume, improvements in skin lesions and pulmonary function. The renal and pulmonary benefits of treatment with everolimus tended to reverse after the drug was withdrawn, though the improvements were persistent in part patients.

In patients with the tuberous sclerosis complex, renal disease is a leading cause of death or disability in adult patients [3]. TSC is a rare autosomal dominant genetic disease caused by mutations in theTSC1 gene coding for hamartin and TSC2 gene coding tuberin $[5,6]$. Finding that tuberin plays an important role in mTOR signaling pathway and further identification of tuberin hamartin complex as a main inhibitor of this pathway opened up new possibilities in disease-modifying therapy for TSC patients $[12,13]$. Randomized clinical trials support the use of everolimus, an inhibitor of the mammalian target of rapamycin, in the treatment of subependymal giant cell astrocytomas (SEGA), RAML and seizure related to TSC in western populations $[8,14,15]$. However, its efficacy and safety in treatment of Chinese TSC patients is unknown. In the trial, tumor partial response by RECIST criteria was observed in 52.94\%, 58.82\% and $66.67 \%$ at months 3, 6 and 12 in Chinese adult patients, respectively. Bissler and colleagues investigated everolimus treatment for RAML in patients with tuberous sclerosis and found $44.2 \%, 55 \%$ and $64.5 \%$ patients achieved $\geq 50 \%$ reduction from baseline in the sum of volumes of target lesions at weeks 12, 24 and 96, respectively [8, 16]. Another subgroup analysis from EXIST-1 trial showed similar results that the proportions of patients in the everolimus arm with 50\% reduction in the sum of target RAML were $56.5 \%, 78.3 \%$ and $80.0 \%$ after 12,24 and 48 weeks, respectively [17]. In addition, Bissler and colleagues investigated another mTOR inhibitor, sirolimus, treatment for RAML in patients with tuberous sclerosis or sporadic LAM and found a mean reduction in RAML volume of $47 \%$ at 12 months [18]. The observation that angiomyolipoma size correlates with the risk of hemorrhage suggests that maintains or reduces angiomyolipoma size may reduce the risk of bleeding. However, the mean angiomyolipoma volume had increased ranging from a rapid return to baseline dimensions to a sustained reduction in size, after the withdrawal of everolimus. The current trial shows that everolimus are effective in reducing RAML size in tuberous sclerosis in Chinese patients. But long-term maintenance of everolimus therapy is necessary in TSC- 
RAML patients as it increased after stopping therapy. Everolimus administration to patients with TSC-RAML over the extension phase of the EXIST-2 trial supports a long-term benefit over approximately 4 years [19]. Therefore, it seems reasonable to assume that TSC-RAML patients should be kept under mTOR inhibition for life.

Six patients with lymphangioleiomyomatosis were evaluated for pulmonary outcomes. After 12 months of everolimus therapy, the FEV1 increased by $276 \mathrm{ml}$, and the FVC increased by $433 \mathrm{ml}$, while the mean residual volume fell by $408 \mathrm{ml}$. In addition, the FEV1, FVC and residual volume remained the most improved 12 months after everolimus was stopped, as compared with the baseline values. However, neither the DLco nor total lung capacity changed significantly during everolimus therapy. In addition, facial angiofibromas decreased in size and became paler and less rough after 12 months of therapy, which remained the most improved 12 months after stopping everolimus. There was no change in subependymal nodules size during the study. However, everolimus appears to have activity in the central nervous system, on the basis of a slight improvement in the frequency of seizures was reported by two patients. In all, treatment with everolimus for 1 year resulted in an improvement in lung function and skin lesions in adults with the TSC-RAML.

Adverse events were common, consistent with the known toxicities of everolimus and mostly of low grade. The most frequent adverse reactions we recorded were mucositis oral (100\%), which was observed at the start of treatment. The most common grade 3 adverse events were irregular menstruation, occurred in five out of twelve female patients (25.0\%). The frequency of mucositis oral and irregular menstruation was higher than the EXIST-2 and subgroup analysis of EXIST-1 study [1, 3], which may be due to the different race of patients. Although three cases of amenorrhoea in our study resolved without intervention, surveillance for this potential side effect is warranted in women patients of child-bearing potential and needed further investigated. In the current trial, one patient died from RAML spontaneous haemorrhage during treatment with everolimus, even with reduction in RAML volume of $60.68 \%$ at 3 months. As far as we know, it is the first case reported RAML spontaneous haemorrhage during treated with everolimus. The sum of RAML volume burben is over $4000 \mathrm{ml}$ in this patient, which may the major reason for RAML spontaneous haemorrhage. A second death was due to epithelioid RAML progression, with metastasis to multiple retroperitoneal lymph node, and died from severe infection one month after surgery. Epithelioid RAML is considered to be a potentially malignant tumor, which is composed of a prominent epithelioid component, with spindle and giant cells, and contains none or a minimal amount of adipose tissue. Epithelioid RAML can occur in patients both with and without tuberous sclerosis. However, half of published cases have history of tuberous sclerosis, some show metastatic potential $[20,21]$. In addition, the mTOR pathway was recently found to be activated in epithelioid angiomyolipoma [22], and Wolff $\mathrm{N}$, et al. reported that mTOR inhibitors, such as sirolimus or temsirolimus, in two cases of epithelioid angiomyolipoma and showed a good short term response [23]. The two patients died during the clinical trial, reminds us that the risks and benefits of everolimus need specific and careful evaluation in the real world.

Several limitations in our study should be noted: (1) it is a single center based on a small sample without placebo-controlled trial; (2) none patient with TSC1 gene mutation was included; (3) all patients were adult, so clinical trials concerning the safety of everolimus for child patients needed in the future.

\section{Conclusions}

Collectively, the data suggest that everolimus therapy for 1 year not only resulted in a decrease in the size of angiomyolipomas, but also improved in lung function and skin lesions in adults Chinese patients with TSC-RAML. One year after the drug was discontinued, the angiomyolipoma size and the lung function approached, but did not completely return to, the baseline values. It seems reasonable to assume that TSC-RAML patients should be kept under mTOR inhibition for life. The most common adverse events were mucositis oral, irregular menstruation, abdominal pain, hypertriglyceridemia and headache. The two patient who died in the current trial reminds us that the risks and benefits of everolimus need specific and careful evaluation in the real world. In conclusion, this study shows that everolimus is a relatively safe and efficacious in treatment of TSC-RAML in Chinese adult patients.

\section{Additional files}

Additional file 1: Visit Schedule and Assessments. (DOCX $73 \mathrm{~kb}$ )
Additional file 2: Physician's Global Assessment of Clinical Condition.
(DOCX $98 \mathrm{~kb}$ )

Additional file 3: Mutations detected by next-generation sequencing. (DOCX $92 \mathrm{~kb}$ )

\section{Abbreviations \\ CT: Computed tomography; DLCo: Denotes diffusing capacity of the lung for carbon monoxide; FEV1: Forced expiratory volume in $1 \mathrm{~s}$; FVC: Forced vital capacity; LAM: Lymphangioleiomyomatosis; MRI: Magnetic resonance imaging; mTOR: Mammalian target of rapamycin; RAML: Renal angiomyolipoma; SEGA: Subependymal giant cell astrocytomas; TSC: Tuberous sclerosis complex}

\section{Acknowledgements}

We thank the personnel at the department of Dermatology, Peking Union Medical College Hospital and the personnel the department of Respiratory Medicine, Peking Union Medical College Hospital for their support.

\section{Funding}

The research was supported by Novartis Pharmaceuticals Corporation (10288), the National Natural Science Foundation of China (81670611) and 
the the National Key Research and Development Program of China (2016YFC0901500)

\section{Availability of data and materials}

Data are available on request.

\section{Authors' contributions}

YSZ had full access to all the data in the study and takes responsibility for the integrity of the data and the accuracy of the data analysis. Study concept and design: YSZ, HZL. Acquisition of data: YC, HG, WDW, HS, BS. Analysis and interpretation of data: YC, HG. Drafting of the manuscript: YC, HG, WDW. Critical revision of the manuscript for important intellectual content: YSZ, HZL. Statistical analysis: YC, HG, YSZ. Obtaining funding: YSZ. Administrative, technical, or material support: YSZ. All authors read and approved the final manuscript.

\section{Ethics approval and consent to participate}

All patients provided written informed consent before entering the clinical trial. The study was done in accordance with the Declaration of Helsinki and local regulations This study protocol was approved by the Human Ethics Committee of Peking Union Medical College Hospital, before the first patient was enrolled.

\section{Consent for publication}

Not applicable.

\section{Competing interests}

Yi Cai received travel expenses from Novartis CO., LTD. The other authors declare that they have no competing interests.

\section{Publisher's Note}

Springer Nature remains neutral with regard to jurisdictional claims in published maps and institutional affiliations.

\section{Author details}

${ }^{1}$ Department of Urology, Peking Union Medical College Hospital, Chinese Academy of Medical Sciences and Peking Union Medical College, 1 Shuaifuyuan Road, Beijing 100730, China. ${ }^{2}$ Department of Radiology, Peking Union Medical College Hospital, Chinese Academy of Medical Sciences and Peking Union Medical College, Beijing 100730, China.

\section{Received: 13 December 2017 Accepted: 8 March 2018}

\section{Published online: 27 March 2018}

\section{References}

1. Northrup H, Krueger DA. Tuberous sclerosis complex diagnostic criteria update: recommendations of the 2012 linternational tuberous sclerosis complex consensus conference. Pediatr Neurol. 2013;49(4):243-54.

2. Cai Y, Li H, Zhang Y. Assessment of tuberous sclerosis complex associated with renal lesions by targeted next-generation sequencing in mainland China. Urology. 2017;101:170.e1-7.

3. Kapoor A, et al. Evolving strategies in the treatment of tuberous sclerosis complex-associated Angiomyolipomas (TSC-AML). Urology. 2016;89:19-26.

4. Ting WY, et al. Clinical analysis of tuberous sclerosis complex complicated with renal angiomyolipoma: a report of 22 cases. Zhonghua Yi Xue Za Zhi. 2013;93(26):2056-8.

5. Consortium ECTS. Identification and characterization of the tuberous sclerosis gene on chromosome 16. Cell. 1993;75(7):1305-15.

6. van Slegtenhorst $M$, et al. Identification of the tuberous sclerosis gene TSC1 on chromosome 9q34. Science. 1997:277(5327):805-8.

7. Laplante M, Sabatini DM. mTOR signaling in growth control and disease. Cell. 2012;149(2):274-93.

8. Bissler JJ, et al. Everolimus for angiomyolipoma associated with tuberous sclerosis complex or sporadic lymphangioleiomyomatosis (EXIST-2): a multicentre, randomised, double-blind, placebo-controlled trial. Lancet. 2013;381(9869):817-24.

9. Krueger DA, Northrup H. Tuberous sclerosis complex surveillance and management: recommendations of the 2012 international tuberous sclerosis complex consensus conference. Pediatr Neurol. 2013:49(4):255-65.

10. Duvic M, et al. Phase 2 and 3 clinical trial of oral bexarotene (Targretin capsules) for the treatment of refractory or persistent early-stage cutaneous T-cell lymphoma. Arch Dermatol. 2001;137(5):581-93.
11. Heald $P$, et al. Topical bexarotene therapy for patients with refractory or persistent early-stage cutaneous T-cell lymphoma: results of the phase III clinical trial. J Am Acad Dermatol. 2003;49(5):801-15.

12. Gao X, et al. Tsc tumour suppressor proteins antagonize amino-acid-TOR signalling. Nat Cell Biol. 2002;4(9):699-704.

13. Tee $A R$, et al. Tuberous sclerosis complex-1 and -2 gene products function together to inhibit mammalian target of rapamycin (mTOR)-mediated downstream signaling. Proc Natl Acad Sci U S A. 2002;99(21):13571-6.

14. French JA, et al. Adjunctive everolimus therapy for treatment-resistant focal-onset seizures associated with tuberous sclerosis (EXIST-3): a phase 3, randomised, double-blind, placebo-controlled study. Lancet. 2016; 388(10056):2153-63.

15. Franz DN, et al. Efficacy and safety of everolimus for subependymal giant cell astrocytomas associated with tuberous sclerosis complex (EXIST-1): a multicentre, randomised, placebo-controlled phase 3 trial. Lancet. 2013; 381(9861):125-32

16. Bissler JJ, et al. Everolimus for renal angiomyolipoma in patients with tuberous sclerosis complex or sporadic lymphangioleiomyomatosis: extension of a randomized controlled trial. Nephrol Dial Transplant. 2016;31(1):111-9.

17. Kingswood JC, et al. The effect of everolimus on renal angiomyolipoma in patients with tuberous sclerosis complex being treated for subependymal giant cell astrocytoma: subgroup results from the randomized, placebocontrolled, phase 3 trial EXIST-1. Nephrol Dial Transplant. 2014;29(6):1203-10.

18. Bissler JJ, et al. Sirolimus for angiomyolipoma in tuberous sclerosis complex or lymphangioleiomyomatosis. N Engl J Med. 2008;358(2):140-51.

19. Bissler JJ, et al. Everolimus long-term use in patients with tuberous sclerosis complex: four-year update of the EXIST-2 study. PLoS One. 2017;12(8): e0180939.

20. Lopez-Beltran A, et al. 2004 WHO classification of the renal tumors of the adults. Eur Urol. 2006;49(5):798-805.

21. Lane BR, et al. Clinical correlates of renal angiomyolipoma subtypes in 209 patients: classic, fat poor, tuberous sclerosis associated and epithelioid. J Urol. 2008;180(3):836-43.

22. Pan CC, et al. Constant allelic alteration on chromosome $16 \mathrm{p}$ (TSC2 gene) in perivascular epithelioid cell tumour (PEComa): genetic evidence for the relationship of PEComa with angiomyolipoma. J Pathol. 2008;214(3):387-93.

23. Wolff $\mathrm{N}$, et al. Sirolimus and temsirolimus for epithelioid angiomyolipoma. J Clin Oncol. 2010;28(5):e65-8.

\section{Submit your next manuscript to BioMed Central and we will help you at every step:}

- We accept pre-submission inquiries

- Our selector tool helps you to find the most relevant journal

- We provide round the clock customer support

- Convenient online submission

- Thorough peer review

- Inclusion in PubMed and all major indexing services

- Maximum visibility for your research

Submit your manuscript at www.biomedcentral.com/submit
) BioMed Central 